\title{
Postoperative grey-white lines of the posterior cornea and endothelial cell damage
}

\author{
HANIA C LAGANOWSKI, MALCOLM G KERR MUIR, \\ AND EMIL S SHERRARD
}

From the Department of Clinical Ophthalmology, Institute of Ophthalmology, and Moorfields Eye Hospital, City Road, London EC

SUMMARY 'Snailtracks' (grey-white streaks and patches on the posterior corneal surface which occur postoperatively) have recently been implicated as possible markers of corneal endothelial cell damage. We have examined this phenomenon in vivo with the slit-lamp and specular microscope. We recognised three types of linear form and, in contradiction of other observers, found that each was always coincident with a wrinkle in Descemet's membrane. Moreover, we were able to demonstrate only minimal endothelial cell damage in only a few of the tracks.

It seems probable that grey-white lines at the level of the corneal endothelium are a frequent phenomenon following extracapsular cataract extraction (ECCE) with or without intraocular lens (IOL) implantation, and perhaps other intraocular procedures. However, it appears that little regard has been paid to them, if and when noted, probably because of their transience and apparent lack of long-term significance.

Alfonso $e t$ al.' have recently drawn attention to these changes, calling them 'snailtracks' because of their slit-lamp appearance - that is 'irregular, discontinuous, greyish-white streaks or patches, usually oriented horizontally and obliquely, but also seen oriented vertically'.

On specifically seeking snailtracks with the slitlamp these authors noted them on the first postoperative day in 42 out of $46(91 \%)$ consecutive patients undergoing ECCEs. In order to determine the precise nature of the snailtracks they examined by scanning electron microscopy similar 'lesions' noted in a corneal button stored in McCarey-Kaufman medium and those induced in a human cadaver eye by ECCE. They found a gradation of endothelial cell changes culminating in irreversible cell damage and sloughing from Descemet's membrane. On this evidence they suggested that the presence of snailtracks in in-vivo eyes may 'have clinical relevance as a marker for corneal endothelial cell damage'.

The purpose of this study was to investigate further

Correspondence to Dr E S Sherrard, Professorial Unit, Moorfields Eye Hospital, City Road, London EC1V 2PD. the precise nature and significance of snailtracks and to support or otherwise the above contention of Alfonso et al. If snailtracks are markers of endothelial loss, they become of increasing concern as more ECCEs with or without IOL implantation are performed in young people, since early, acute loss of endothelial cells with consequent, subsequent accelerated age-related loss $^{2}$ may result in corneal decompensation later in life.

To eliminate the uncertainties of extrapolation from the in-vitro to the in-vivo state snailtracks of the corneal endothelium were studied with the slit-lamp and high magnification specular microscope in the living human eye. No attempt was made to determine their aetiology.

\section{Material and methods}

SLIT LAMP EXAMINATION

Snailtracks as defined by Alfonso et al.' were observed by careful slit-lamp examination in 20 patients after ECCE with IOL implantation. To determine their general characteristics various methods of illumination were used-for example, direct, retro-, and specular. In order to seek and examine vertically orientated snailtracks with the horizontal axis of the cornea parallel with the vertical slit-beam one patient was examined with her head sideways so that the vertical axis of the eye was horizontal.

The examinations were performed on the first and 
second postoperative days in all cases, and additionally on the fourteenth day in 10 cases.

\section{SPECULAR MICROSCOPY}

Following slit-lamp examination on the first postoperative day the endothelia of six of the cases were examined and photographed with the large-field specular microscope (Pocklington, Keeler/Konan). These same areas of endothelia were relocated, by reference to the posterior corneal rings, ${ }^{3}$ and examined on various subsequent postoperative days as indicated by the slit-lamp appearances.

\section{MODEL}

To aid the interpretation of the nature of snailtracks a simple model was constructed to mimic the posterior surface of the cornea.

A single layer of transparent polyethylene Clingfilm was mounted over the mouth of a $20 \mathrm{~mm}$ diameter hollow cylinder such that it could be blown into a convex shape by air pressure from behind. Wrinkles were then induced in it by withdrawing a small volume of air. The wrinkled polyethylene was carefully examined with the slit-lamp.

\section{Results}

\section{SLIT-LAMP APPEARANCE}

Streak-like or linear tracks (Fig. 1) were found in all corneas examined with the slit-lamp on the first postoperative day. Their numbers varied from eye to eye and in general they were concentrated in the upper one-third to one-half of the cornea.

The intensity of horizontally and obliquely orientated linear tracks was variable. In some eyes some or all of them were extremely obvious and clearly visible with almost any setting of the slit-lamp, while in others they were very faint and could easily have been overlooked by the casual observer.

In some cases vertical tracks, although often vague, were also seen, and the result was an overall criss-cross pattern. When the cornea was examined with the horizontal axis of the eye parallel to the vertical slit beam, tracks with vertical orientation relative to the eye became more obvious than those with other orientations. This suggested that the apparent infrequency and indistinctness of vertical tracks is a function of illumination.

The linear tracks showed straight, curved, and even hooked forms and were either continuous or segmented (Fig. 1).

They measured from approximately $0.1 \mathrm{~mm}$ to $8 \mathrm{~mm}$ in length and $0.1 \mathrm{~mm}$ to $0.5 \mathrm{~mm}$ in width, as determined from slit-lamp photographs.

Many of the tracks had a distinctly granular appearance and irregular edges (Fig. 1). They either appeared white and suggested endothelial changes, or had a yellowish tinge with scattered pigment suggesting the deposition of fine particulate matter (which was observed circulating in the aqueous humour in all eyes). Other tracks were grey and agranular with straight margins. In many cases the appearance of a single track varied along its length with white-granular and agranular segments.

It was found that the use of a broad slit beam at right angles to the axes of the viewing system and of the forward gazing eye revealed the linear tracks of all types to the best effect (Fig. 1). When the area of the cornea in view was illuminated by a beam directed more or less parallel to its surfaces, these tracks appeared as described. However, when the same area was illuminated at right angles to its surfaces, the tracks appeared as wrinkles in the posterior cornea, and small patches of specular reflection from their edges were evident-usually where two tracks at different orientations crossed. By changing from one direction of illumination to another it could always be shown that linear tracks of all orientations were wrinkles and vice versa (Fig. 2 a, b).

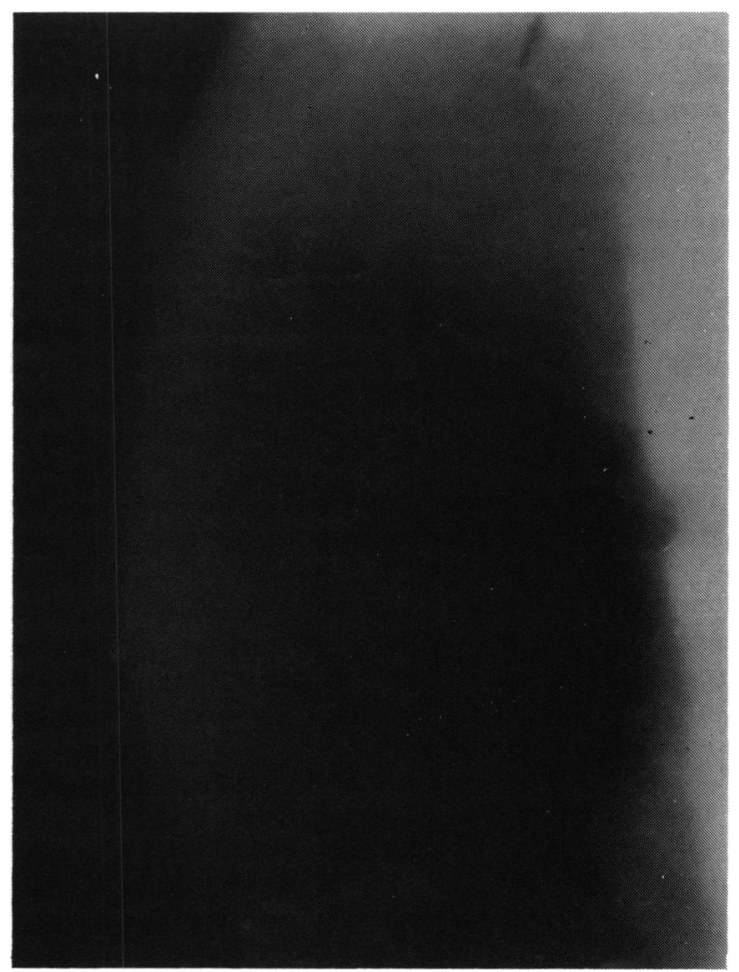

Fig. 1 Slit-lamp photograph, with broad beam illumination at $90^{\prime}$, of cornea showing granular, linear tracks one day after ECCE/IOL 
Fig. 3 a

Fig. 3 Specular photomicrograph of endothelium one day after ECCE/IOL. Bar $=0 \cdot 1 \mathrm{~mm}$. (a) With normal pressure of applanation-excessive wrinkles are present. (b) Same area as (a) with greater pressure of applanation-most of the wrinkles are no longer apparent. (Arrows indicate same cell in $(a)$ and $(b)$.

Fig. 2 Slit-lamp photographs of cornea with linear tracks. (a) With illumination parallel to corneal surfaces the tracks appear as greyish-white lines. (b) Same area as (a) with illumination at right angles to corneal surfaces the tracks appear as wrinkles in the posterior cornea. (Arrows indicate same point in (a) and (b).

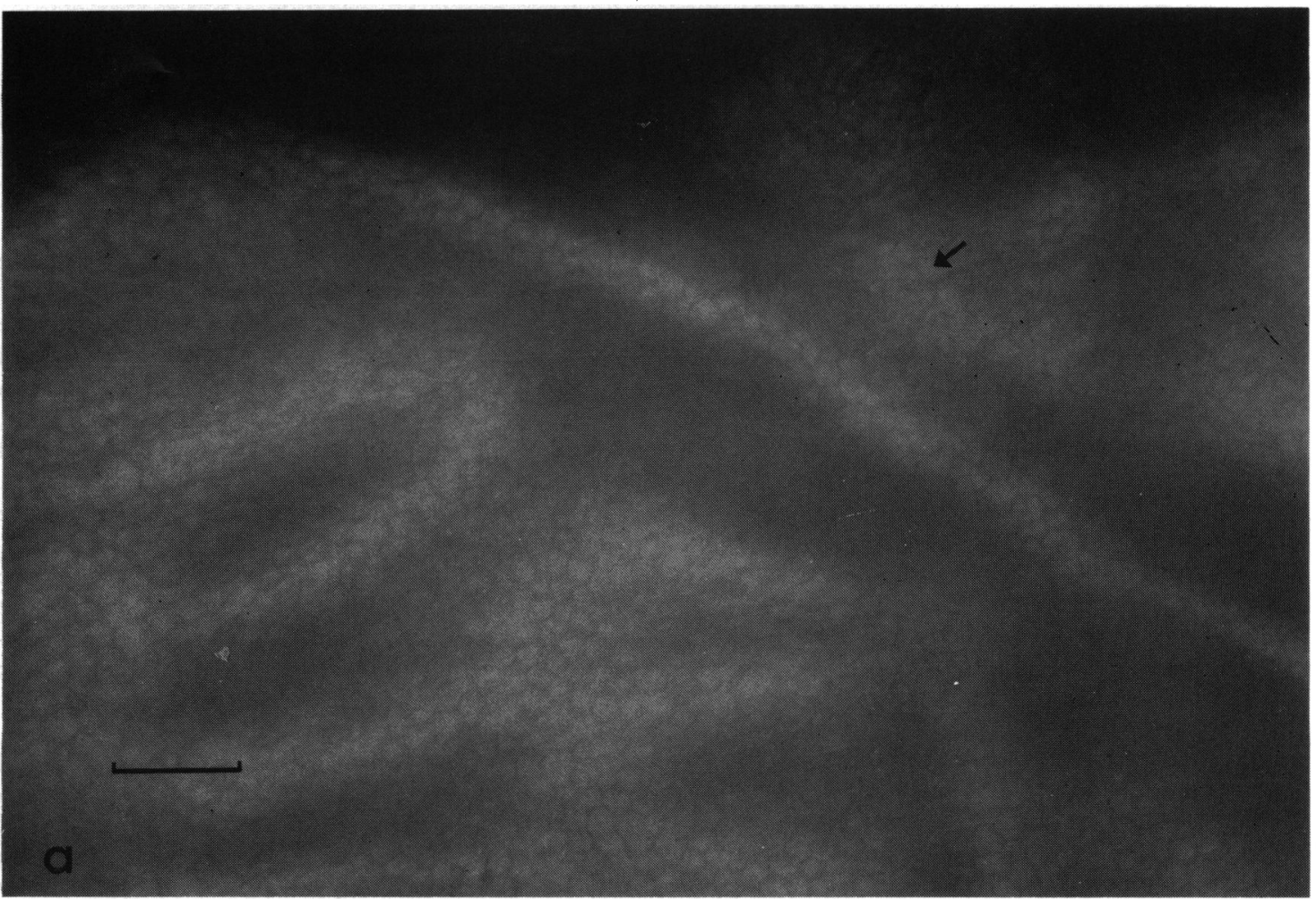




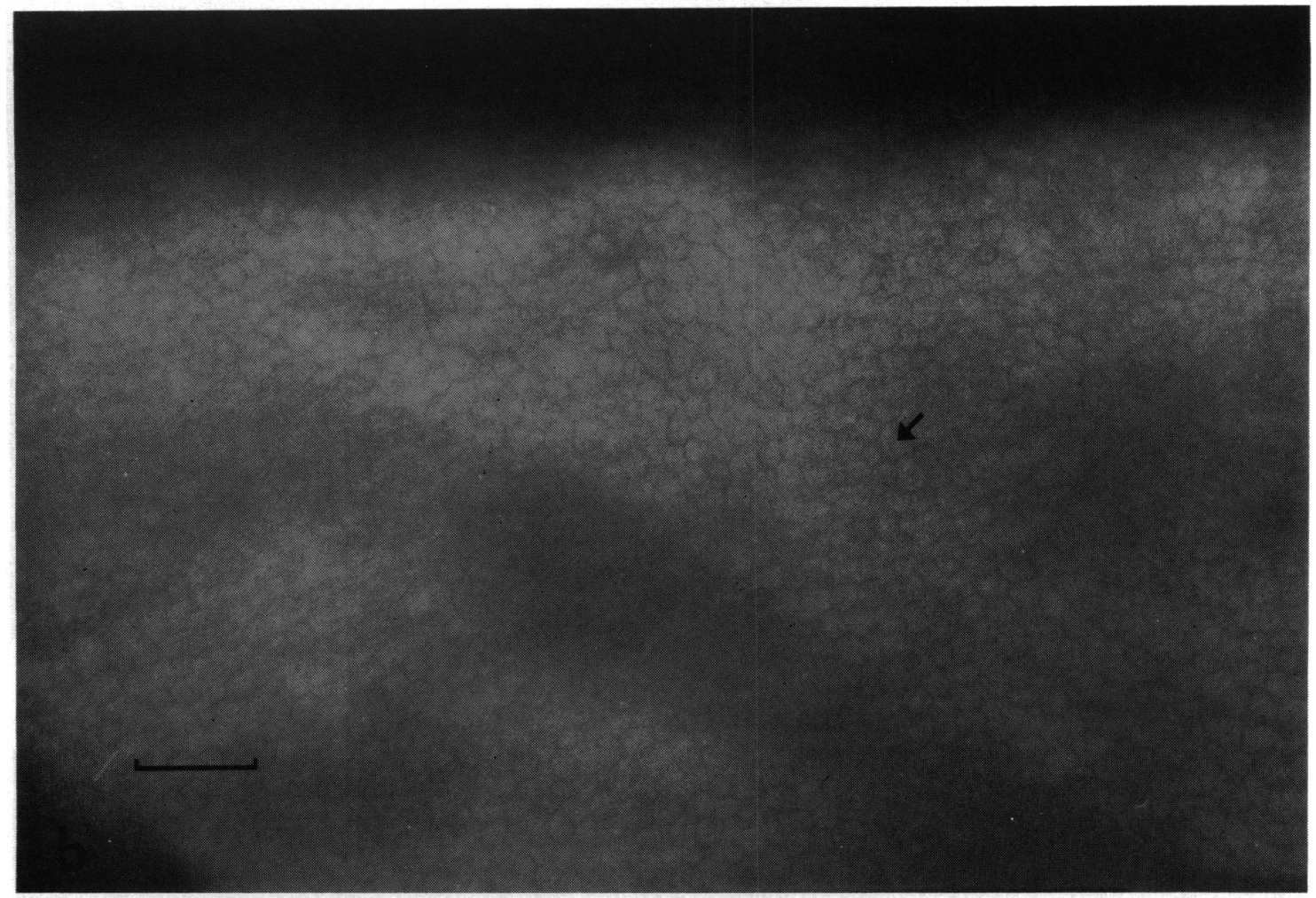

Fig. $3 \mathrm{~b}$

In many instances there were a few blotch-like patches, distinct from the linear tracks, on the posterior corneal surface. These had a similar 'texture' to the yellow-tinted, linear tracks and an appearance suggesting deposition of particulate matter. They were not coincident with detectable distortions of Descemet's membrane.

On the second postoperative day there was little change in the tracks in many cases. However, some of the previously elongated granular forms had broken up into irregular segments separated by agranular sections, and in a few cases only small, scattered, opaque segments remained. Many of the blotch-like patches had disappeared.

Tracks were still present in three of the 10 eyes two weeks postoperatively.

\section{MODE L}

On examining the polyethylene model with direct illumination and viewing, grey-white lines similar to agranular linear tracks were visible in the otherwise transparent membrane. However, on moving the model relative to the slit-lamp the true nature of these lines - that is, the sloping sides of wrinkleswas easily demonstrated by achieving specular reflection from their edges and troughs or crests.

\section{SPECULAR MICROSCOPY}

In all six eyes examined with the specular miscoscope on the first postoperative day excessive numbers of shallow wrinkles of the posterior corneal surface were seen (Fig. 3a). These were consistent in both number and orientation with the linear tracks seen with the slit-lamp on the same occasion. There were, however, surprisingly few instances of endothelial change or particulate deposits associated with them. This might have been because any changes were hidden in the dark area of the wrinkles (Fig. 3a). However, it was possible in most cases to flatten some of the wrinkles by applying more pressure with the applanating objective, and render visible the endothelium previously incorporated in them; this revealed normal endothelium in most instances (Fig. 3b).

Where changes were observed, the endothelial mosaic was greatly distorted along much of the length of the wrinkle. This was mainly due to elongation of the cells in two or three rows, with the long axes of the cells orientated obliquely towards the long axis of the wrinkle, giving a pinnate arrangement (Fig. 4). Along some wrinkles there were groups of elongated cells radiating from a point which were separated by normal cells. 


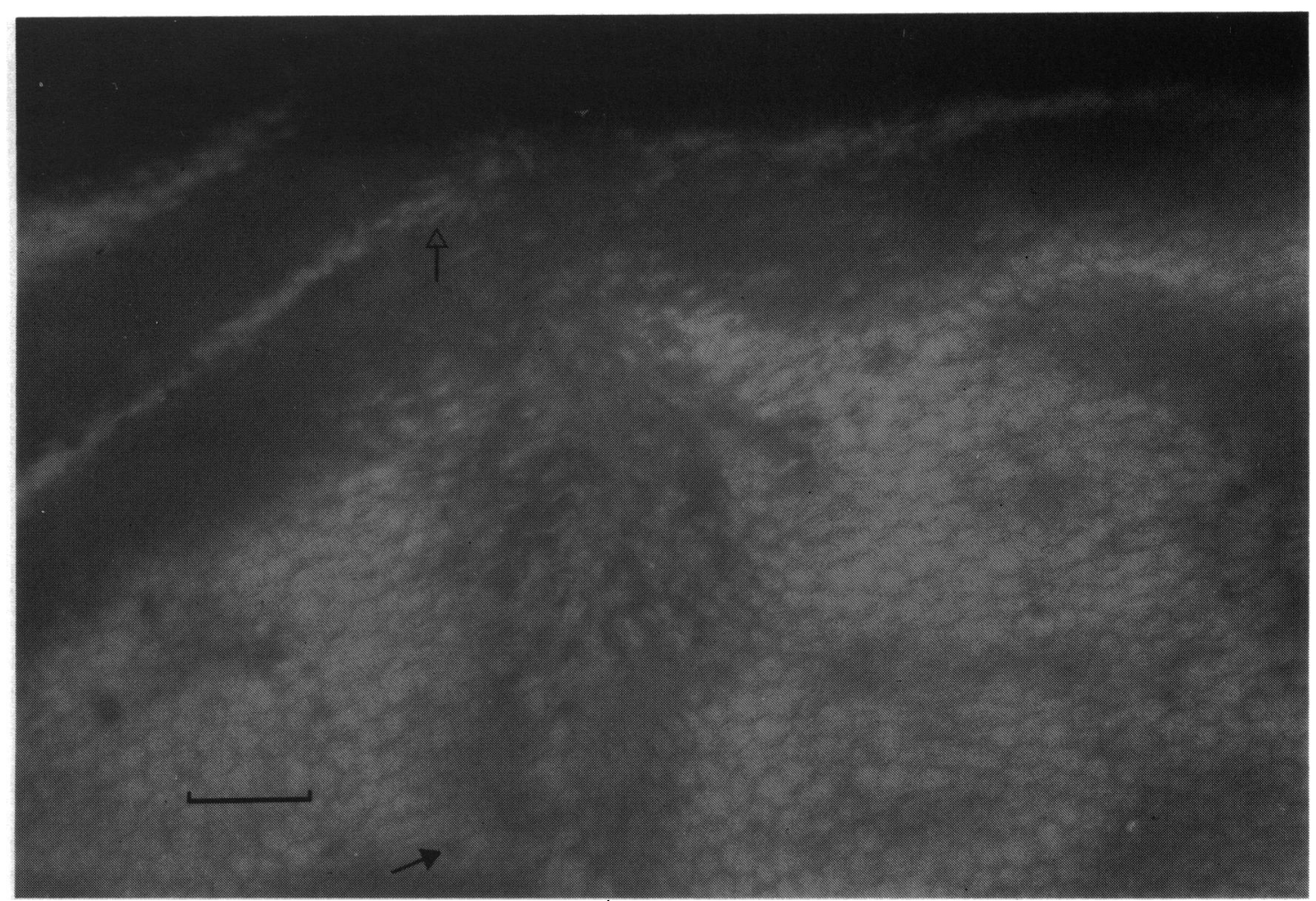

Fig. 4 Specular photomicrograph of endothelium one day after ECCE/IOL showing pinnate arrangement of elongated cells along a vertical wrinkle. A rosette (solid arrow) and a group of spindle-shaped cells (open arrow) are present. Bar $=0.1 \mathrm{~mm}$.

In two eyes similar but non-linearly distributed changes were seen which were not associated with wrinkles (Fig. 5).

On a smaller scale rosettes (a group of neighbouring cells elongated to a common point) were numerous within the altered areas (Figs. 4, 5). There were occasional groups of thin spindle-like cells (Fig. 4) and many cells possessed inclusions-probably vacuoles (Fig. 5).

Away from the altered areas, even within the wrinkles, the endothelium showed normal polymegethism in four of the eyes, and was composed of slightly enlarged regular cells in the other two.

The specular microscope, in either the specular or the relief mode,${ }^{+}$failed to demonstrate the presence of fine granular deposits in linear form, but in some cases generalised deposition of fine material in the entire lower half of the cornea was noted in the relief mode.

On the second postoperative day wrinkles were observed as before but were more easily flattened. Along the course of those wrinkles which had previously shown distortion of the endothelial mosaic there were discontinuous areas containing endo- thelial cells of bizarre shapes and sizes, many being larger than those found elsewhere. Rosettes were more common than on the previous day, but only a few cells now possessed inclusions. Between the patches of deformed cells, but still along the course of the wrinkle, the endothelium appeared normal.

In the one case examined with the specular microscope 14 days postoperatively no endothelial abnormality was detected on any of the flattened wrinkles or elsewhere, even though limited cell damage had been seen on the first postoperative day.

\section{Discussion}

Greyish white streaks and patches at the level of the endothelium, which by definition are 'snailtracks', were seen in all the corneas after ECCE with IOL implantation, although their number, pattern, and character varied.

Alfonso et al. ${ }^{\prime}$ made the unsupported statement that 'snailtracks can be easily differentiated from Descemet's folds'. Our slit-lamp examinations showed that linear tracks were always coincident with wrinkles (small folds) and only the blotch-like 


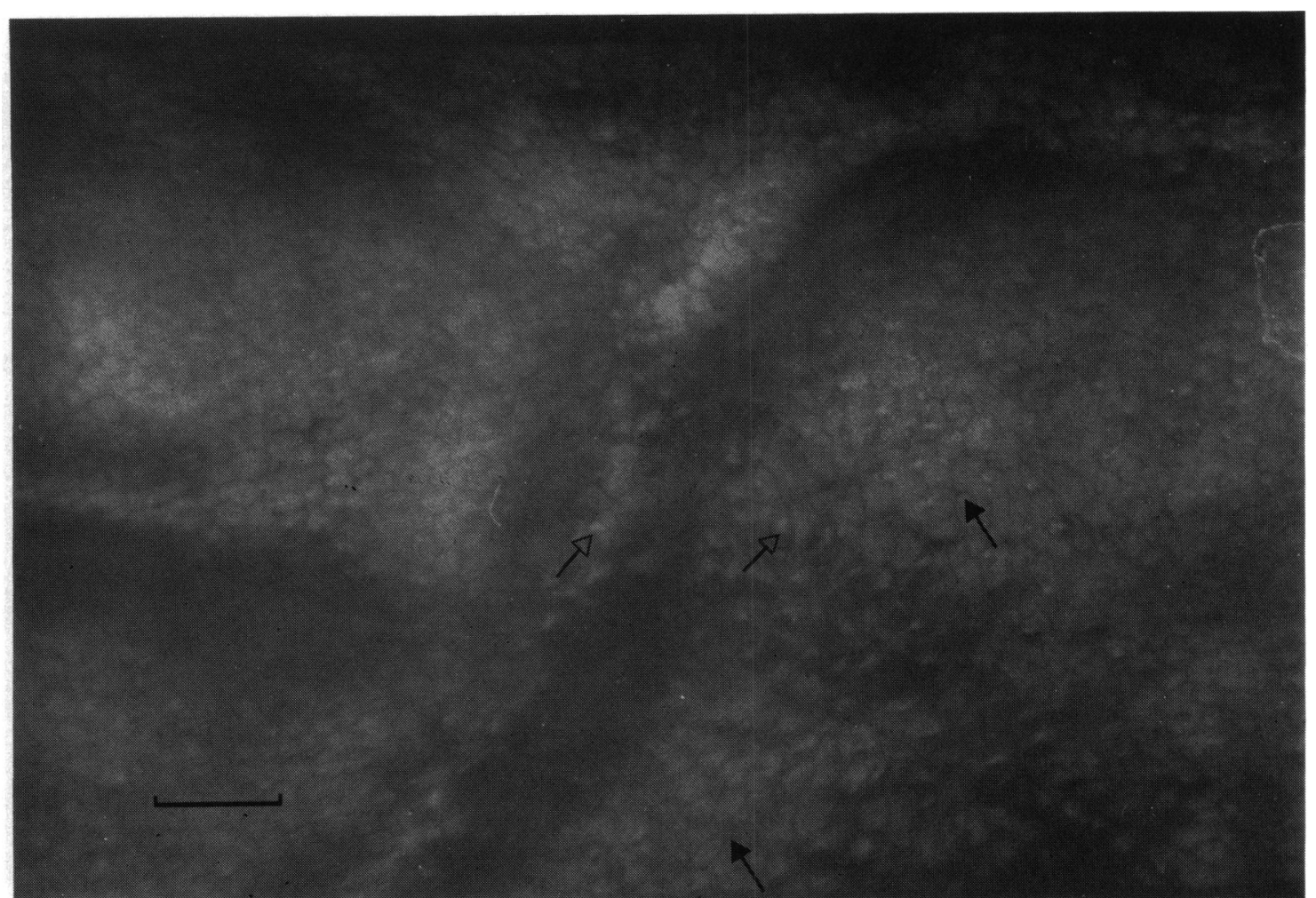

Fig. 5 Specular photomicrograph of endothelium one day after ECCE/IOL showing non-linear area of altered cells, rosettes (solid arrows), and cells with inclusions (open arrows). Bar $=0.1 \mathrm{~mm}$.

patches (which we note are included in the original description of 'snailtracks') were not. Whereas in some cases it was obvious that the tracks were wrinkles, in others this was difficult to demonstrate, but was consistently confirmed by achieving specular reflection from the edges of the wrinkles. Specular microscopy confirmed that the linear tracks were wrinkles.

It is our contention that all linear tracks are small wrinkles of Descemet's membrane. Because of their extreme shallowness the slit-lamp appearance of greyish white lines dominates, and the presence of a wrinkle often becomes apparent only on achieving specular reflection from its edges and trough or crest. In the case of large wrinkles the reverse is true; the wrinkles are obviously wrinkles and it is necessary to 'become aware' that their sloping sides actually appear as greyish white bands.

As in the polyethylene model, some of the wrinkles appeared with direct illumination as greyish white, otherwise featureless streaks. Others had a definite granular appearance, and two types were recognisable - white, which suggested damaged endothelial cells, and yellow tinted, which looked like adherent debris.
On rare occasions specular microscopy revealed endothelial changes which in some cases were consistent with irreversible cell damage. It has been shown experimentally that a rosette results from the loss of a single cell and that cells around the void extend processes into it to re-establish continuity of the endothelium within a matter of hours. ${ }^{5}$ Spindlelike rosettes have been seen within hours of known cell loss resulting from Nd/YAG laser procedures." Intracellular vacuoles are a sign of current disturbance of affected cells. ' Enlargement and distortion of cells is considered a late sign of cell loss. ${ }^{\times}$

Specular microscopy did not confirm the slit-lamp impression of localised deposition of fine debris, either in linear or in patch form.

While it is tempting to conclude that (1) the simple wrinkles without associated endothelial change, as seen with the specular microscope, are those tracks that appear agranular with the slit-lamp, (2) those with endothelial damage are granular tracks, (3) those with discontinuous areas of endothelial damage are segmented tracks, and (4) the areas of endothelial change not associated with a wrinkle are the blotchlike patches, these relationships could not be con- 
firmed, because no track of any particular slit-lamp appearance was knowingly examined by specular microscopy. Nevertheless, some of the corneas examined with the specular microscope were known to have a predominance of granular tracks, and there was therefore a strong probability that many of the wrinkles seen by specular microscopy were granular tracks, and yet only occasionally was endothelial damage found.

In general our slit-lamp impressions did not correlate with our specular microscopical findings, possibly because either our interpretations of the slitlamp images were incorrect or the specular microscope failed to resolve the features they suggested.

Although our in-vivo observations have done little to elucidate the true nature of snailtracks, there is no doubt that endothelial damage is present in parts of some of them. However, because the majority of tracks show normal endothelium, they cannot be regarded as markers of endothelial cell damage.

While the linear tracks are basically wrinkles, the blotch-like forms are not associated with any detectable distortion of Descemet's membrane and they cannot therefore be regarded as the same entity. Moreover, in our experience snails are not prone to creating random blotch-like tracks and we would therefore favour reserving the term 'snailtrack' as descriptive of the linear forms only.

\section{References}

1 Alfonso E. Tucker GS, Batlle JF, Mandelbaum S, Gelender H. Forster RK. Snailtracks of the corneal endothelium. Ophthalmology 1986; 93: 344-9.

2 Mishima S. Clinical investigations on the corneal endothelium. Ophthalmology 1982; 89: 525-30.

3 Sherrard ES, Buckley RJ. Relocation of specific endothelial features with the clinical specular microscope. Br J Ophthalmol $1981 ; 65: 820-7$.

4 Sherrard ES, Buckley RJ. The relief mode: a new application of the clinical specular microscope. Arch Ophthalmol 1982; 100: 296-300).

5 Sherrard ES. The corneal endothelium in vivo: its response to mild trauma. Exp Eye Res 1976; 22: 347-57.

6 Kerr Muir MG, Sherrard ES. Damage to the corneal endothelium during Nd/YAG photodisruption. Br J Ophthalmol 1985; 69: $77-85$.

7 Sherrard ES. Characterization of changes observed in the corneal endothelium with the specular microscope. Invest Ophthalmol Vis Sci 1978; 17: 322-6.

8 Laing RA. Sandstrom MM, Berrospi AR. Leibowitz HM. Morphological changes in corneal endothelial cells after penetrating keratoplasty. A m J Ophthalmol 1976; 82: 459-64.

Accepted for publication 1.3 November 1986. 